\title{
Current-Driven Magnetoresistance Oscillations in Asymmetric Spin Valves
}

\author{
M. GMitra ${ }^{a, *}$ AND J. BARNAŚs ${ }^{b}$
}

${ }^{a}$ Department of Theoretical Physics and Astrophysics, P.J. Šafárik University, Park Angelinum 9, 04001 Košice, Slovak Republic and Institute for Theoretical Physics, University of Regensburg 93040 Regensburg, Germany

${ }^{b}$ Department of Physics, Adam Mickiewicz University

Umultowska 85, 61-614 Poznań, Poland

and Institute of Molecular Physics, Polish Academy of Sciences

Smoluchowskiego 17, 60-179 Poznań, Poland

Within the macroscopic model we have calculated spin-transfer torque in asymmetric spin valves in the diffusive transport limit.Such systems exhibit a non-standard angular dependence of the torque, which leads to currentinduced precessional modes at zero external magnetic field. We have found that the frequency of magnetoresistance oscillations exhibits hysteretic behavior when the system is driven between two steady precessional regimes. A finite temperature can suppress the irreversibility and give rise to bistable regime where, both the precessional regimes coexist.

PACS numbers: 75.60.Ch, 75.70.Cn, 75.70.Pa

\section{Introduction}

Transfer of spin angular momentum from conduction electrons to localized magnetic moments in a ferromagnetic body can lead to the phenomenon of currentinduced magnetic switching [1]. The most suitable devices for experimental observation of the effect are spin valves [2]. In typical spin valves the switching between low and high resistive states has been observed for currents exceeding a critical value and for sufficiently low external fields. For larger fields, transition to steady precessional regime can be induced [3]. This behavior is interesting from the application point of view, particularly for designing nanosized dc-current-driven microwave generators. However, it is of great interest to have nanogenerators tuned by current only. The current-driven microwave oscillations in spin-valves

*corresponding author; e-mail: martin.gmitra@upjs.sk 
can be induced if one considers out-of-plane magnetization of the polarizer [4] or an asymmetric spin valve, where a non-standard angular dependence of the torque is induced [5]. In this paper we study the influence of finite temperature and external magnetic field on the frequency of current-driven microwave oscillations.

\section{Model of spin valve}

The spin valve under consideration, $\mathrm{IrMn} / \mathrm{Co}(6) / \mathrm{Ru}(2) / \mathrm{Co}(4) / \mathrm{Cu}(8) /$ $\mathrm{Py}(4) / \mathrm{Cu}$ (where the numbers in parentheses are layer thicknesses in nanometers) contains synthetic antiferromagnetic three-layer $\mathrm{Co}(6) / \mathrm{Ru}(2) / \mathrm{Co}(4)$, coupled via exchange biasing to antiferromagnetic lead, and a permalloy sensing layer. The parallel $(\mathrm{P})$ and antiparallel (AP) configurations refer to the relative alignment of the $\mathrm{Co}(4)$ and $\mathrm{Py}(4)$ layers. The cobalt layers are strongly antiferromagnetically coupled across the Ru layer. Such an arrangement significantly reduces the dipolar field acting on the sensing layer.

To describe the dynamical behavior of the sensing layer we use the LandauLifshitz-Gilbert equation, which additionally includes the torque due to spintransfer. The effective field $\boldsymbol{H}_{\text {eff }}$ acting on the Py layer includes the uniaxial magnetic anisotropy field $H_{\mathrm{a}}$, demagnetization field $\boldsymbol{D}$ of a flat ellipsoid, external field $H_{\mathrm{ext}}$, and the dipolar field $H_{\mathrm{dip}} ; \boldsymbol{H}_{\mathrm{eff}}=-H_{\mathrm{a}}\left(\hat{\boldsymbol{s}} \cdot \hat{\boldsymbol{e}}_{z}\right) \hat{\boldsymbol{e}}_{z}+\boldsymbol{D}-\left(H_{\mathrm{ext}}-H_{\mathrm{dip}}\right) \hat{\boldsymbol{e}}_{z}$, where $\hat{\boldsymbol{e}}_{z}$ is the unit vector along the $z$ axis (in-plane). The spin-transfer torque has the form $\boldsymbol{\tau}=\boldsymbol{\tau}_{\theta}+\boldsymbol{\tau}_{\varphi}$, with $\boldsymbol{\tau}_{\theta}=\tau_{\theta} \hat{\boldsymbol{e}}_{\theta}$, and $\boldsymbol{\tau}_{\varphi}=\tau_{\varphi} \hat{\boldsymbol{e}}_{\varphi}$, where $\hat{\boldsymbol{e}}_{\theta}$ and $\hat{\boldsymbol{e}}_{\varphi}$ are the unit vectors of a coordinate system associated with the polar $\theta$ and azimuthal $\varphi$ angles describing orientation of the sensing layer spin moment $\hat{s}$ with respect to the spin moment of the reference magnetic layer. The torques $\tau_{\theta}$ and $\tau_{\varphi}$ have been calculated within the macroscopic model [6] andcurrent $I$ is defined as positive when it flows from the sensing layer towards the reference one.

\section{Results and discussion}

The torque $\tau_{\theta}$ exerted on the Py sensing layer is almost two orders of magnitude larger than $\tau_{\varphi}$, and plays a significant role in destabilization of both (P and AP) collinear configurations. Moreover, $\tau_{\theta}$ exhibits a non-standard angular dependence, i.e. it vanishes at a non-collinear configuration, $\theta=\theta_{c}[5]$. Thus for positive current both $\mathrm{P}$ and AP configurations are destabilized when current density exceeds the corresponding critical values. In general, critical currents depend on the Gilbert damping,anisotropy field, demagnetization field, and parameters describing transport properties. If one considers the dependence on applied field only, the critical currents can be expressed in the form, $I_{\mathrm{c}}^{\mathrm{P}} / I_{0}=0.156 \tilde{H}_{\text {ext }}+0.35$, and $I_{\mathrm{c}}^{\mathrm{AP}} / I_{0}=-0.044 \tilde{H}_{\mathrm{ext}}+0.1$, where $I_{0}=10^{8} \mathrm{~A} / \mathrm{cm}^{2}$ and $\tilde{H}_{\text {ext }}=H_{\text {ext }} / H_{0}$ with $H_{0}=1 \mathrm{kOe}$. The $I_{\mathrm{c}}^{\mathrm{P}}$ destabilizes the $\mathrm{P}$ state, and the current exceeding $I_{\mathrm{c}}^{\mathrm{AP}}$ destabilizes the AP state. However, $I_{\mathrm{c}}^{\mathrm{P}}>I_{\mathrm{c}}^{\mathrm{AP}}$ and if one considers $H_{\mathrm{ext}}>-1.25 \mathrm{kOe}$ and $I>I_{\mathrm{c}}^{\mathrm{P}}$, the steady precessional regime can be induced even at zero applied field. 

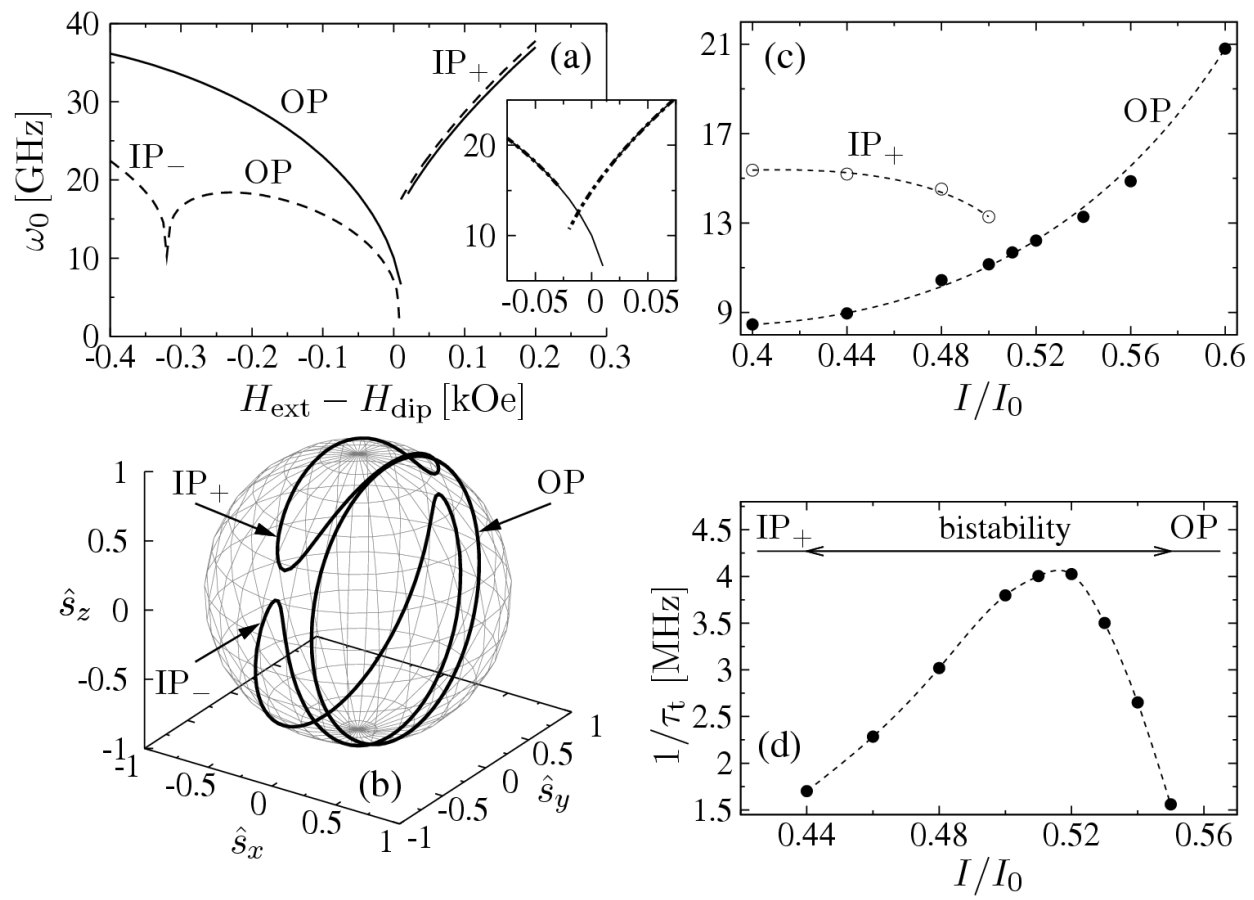

Fig. 1. (a) Current-driven magnetoresistance oscillations as a function of $H_{\text {ext }}-H_{\text {dip }}$ in the zero temperature limit for $I / I_{0}=0.43$ (dashed line) and $I / I_{0}=0.5$ (solid line). The inset shows hysteretic frequency dependence when the field is increased (solid line) and decreased (dashed-dotted line) for $I / I_{0}=0.5$. (b) The corresponding steady state orbits.(c) Frequency $\omega_{0}$ at room temperature as a function of current for $H_{\text {ext }}-H_{\text {dip }}=0$, obtained from the power spectra. (d) Transition rate between $\mathrm{IP}_{+}$and $\mathrm{OP}$ regimes.

The fundamental frequency $\omega_{0}$ of steady oscillations of the parameter $r$, $r=\left[1-\cos ^{2}(\theta / 2)\right] /\left[1+\cos ^{2}(\theta / 2)\right]$, for $I / I_{0}=0.43$ and 0.5 is shown in Fig. 1a as a function of $H_{\text {ext }}-H_{\text {dip }}$. The parameter $r$ describes magnetic configuration of the system and may be different from angular dependence of magnetoresistance. Despite this, we call $r$ reduced magnetoresistance. For $I / I_{0}=0.5$ (solid line), the frequency decreases with increasing $H_{\text {ext }}-H_{\text {dip }}$, and for a small positive value of $H_{\text {ext }}-H_{\text {dip }}$ shows a discontinuity. A further increase in field leads to an increased frequency of the magnetoresistance oscillations. The discontinuity separates the $\mathrm{OP}$ and in-plane $\left(\mathrm{IP}_{+}\right)$regimes. The typical steady oscillatory orbits are shown in Fig. 1b. For $I / I_{0}=0.43$ (dashed line), the frequency first decreases with increasing field, and then increases revealing a profound minimum. The minimum at $H_{\text {ext }}-H_{\text {dip }}=-0.32 \mathrm{kOe}$ separates the in-plane regime close to $-\hat{\boldsymbol{e}}_{z}(\mathrm{IP}-)$ and OP regimes. A further increase in $H_{\text {ext }}-H_{\text {dip }}$ drives the system via the discontinuity in $\omega_{0}$ to the $\mathrm{IP}+$ regime. The presence of the discontinuity gives rise to the hysteretic dependence shown in the inset to Fig. 1a for $I / I_{0}=0.5$. The 
frequency $\omega_{0}$ for the OP regime increases, whereas the frequency for the $\mathrm{IP}_{+}$regime decreases within creasing current. The system exhibits the hysteretic behavior also in the current-driven regime. Thus, both the oscillatory regimes are well separated by irreversible paths.

Finite temperature results in suppresion of the hysteretic behavior due to the finite probability of thermally activated transitions between irreversible states. We have performed simulations at room temperature adding a Langevin random field with Gaussian statistical properties to the effective field $\boldsymbol{H}_{\text {eff }}$. We have found that the hysteresis vanishes due to "telegraph" jumps between the IP + and OP regimes. This appears as a double peak in the power spectra of the magnetoresistance autocorrelation function. The current dependence of $\omega_{0}$, obtained from the spectra for $H_{\text {ext }}-H_{\text {dip }}=0$ is shown in Fig. 1c. The branches for $\mathrm{IP}_{+}$and OP regimes coexist for certain current densities, $0.4<I / I_{0}<0.55$. For increased current, the OP regime dominates the $\mathrm{IP}_{+}$one. The transition frequency $1 / \tau_{\mathrm{t}}$ between both the regimes is shown in Fig. 1d. Thus a finite temperature results in current-driven bistability of the two oscillatory regimes.

\section{Acknowledgments}

This work is partly supported by the Slovak Ministry of Education as a research project MVTS POL/SR/UPJS07 and Polish Ministry of Science and Higher Education as a research project in the years 2006-2009, Slovak Grant Agency VEGA 1/2009/05 as well as Deutsche Forschungsgemeinschaft via SFB 689.

\section{References}

[1] J.C. Slonczewski, J. Magn. Magn. Mater. 159, L1 (1996); 195, L261 (1999); L. Berger, Phys. Rev. B 54, 9353 (1996).

[2] J.A. Katine, F.J. Albert, R.A. Buhrman, E.B. Myers, D.C. Ralph, Phys. Rev. Lett. 84, 3149 (2000). M. Tsoi, V. Tsoi, J. Bass, et al., Phys. Rev. Lett. 89, 246803 (2002).

[3] S.I. Kiselev, J.C. Sankey, I.N. Krivorotov, N.C. Emley, R.J. Schoelkopf, R.A. Buhrman, D.C. Ralph, Nature 425, 380 (2003).

[4] D. Houssameddine, U. Ebels, B. Delat, B. Rodmacq, I. Firastrau, F. Ponthenier, M. Brunet, C. Thirion, J.-P. Michel, L. Prejbeanu-Buda, M.C. Cyrille, O. Redon, B. Dieny, Nature Mater. 6, 447453 (2007).

[5] M. Gmitra, J. Barnaś, Phys. Rev. Lett. 96, 207205 (2006).

[6] J. Barnaś, A. Fert, M. Gmitra, I. Weymann, V.K. Dugaev, Phys. Rev. B 72, 024426 (2005). 